Cinémas

Revue d'études cinématographiques

Revue d'études cinématographiques

Journal of Film Studies

\title{
Du simple au multiple : le cinéma comme série de séries
}

\section{André Gaudreault}

Volume 13, numéro 1-2, automne 2002

Limite(s) du montage

URI : https://id.erudit.org/iderudit/007955ar

DOI : https://doi.org/10.7202/007955ar

Aller au sommaire du numéro

Éditeur(s)

Cinémas

ISSN

1181-6945 (imprimé)

1705-6500 (numérique)

Découvrir la revue

Citer cet article

Gaudreault, A. (2002). Du simple au multiple : le cinéma comme série de séries. Cinémas / Revue d'études cinématographiques, 13(1-2), 33-47.

https://doi.org/10.7202/007955ar

\section{Résumé de l'article}

L'idée de série est au coeur de la problématique de la prise de vues cinématographiques, puisque les " vues animées » sont constituées d'une série de photogrammes (obtenus grâce au procédé du tournage) et que les films sont constitués d'une série de plans (obtenus grâce à la procédure du montage). C'est même par un constant passage dialectique, de l'un au multiple puis du multiple à l'un, que la cinématographie aurait réussi à se développer comme dispositif. Cet article cherche les racines de ce phénomène jusque dans la constitution de la photographie et de la chronophotographie. On y explique comment le passage de la photographie à la chronophotographie est un saut d'ordre quantitatif (production de photographies en plus grand nombre), alors que le passage de cette dernière à la cinématographie relèverait plutôt d'un saut d'ordre qualitatif (plusieurs images fixes qui donnent naissance à une seule image animée). En cinématographie, le dispositif mis en branle permettrait ainsi d'accéder à un seuil d'un autre degré, à un autre ordre de représentation. 


\section{Du simple au multiple: le cinéma comme série de séries}

\section{André Gaudreault}

\section{RÉSUMÉ}

Lidée de série est au cour de la problématique de la prise de vues cinématographiques, puisque les «vues animées» sont constituées d'une série de photogrammes (obtenus grâce au procédé du tournage) et que les films sont constitués d'une série de plans (obtenus grâce à la procédure du montage). C'est même par un constant passage dialectique, de l'un au multiple puis du multiple à l'un, que la cinématographie aurait réussi à se développer comme dispositif. Cet article cherche les racines de ce phénomène jusque dans la constitution de la photographie et de la chronophotographie. On y explique comment le passage de la photographie à la chronophotographie est un saut d'ordre quantitatif (production de photographies en plus grand nombre), alors que le passage de cette dernière à la cinématographie relèverait plutôt d'un saut d'ordre qualitatif (plusieurs images fixes qui donnent naissance à une seule image animée). En cinématographie, le dispositif mis en branle permettrait ainsi d'accéder à un seuil d'un autre degré, à un autre ordre de représentation.

\section{ABSTRACT}

The concept of series lies at the heart of the issue of capturing moving images, since the "animated pictures" are comprised of a series of photograms (obtained through the process of filming) and the films are comprised of a series of shots (obtained through the procedure of editing). Indeed, cinematography succeeded in developing as a dispositif due to the constant dialectical passage from singularity to multiplicity and 
vice versa. This article seeks to uncover the roots of this phenomenon, delving into the formation of photography and chronophotography. We explain how the passage from photography to chronophotography was a quantitive leap (producing a larger number of photographs), while the passage from chronophotography to cinematography was a qualitative leap (many still images giving rise to one single moving image). In cinematography, the dispositif adopted at the outset would result in a shift to a higher level and to another order of representation.

La cinématographie est, à n'en pas douter, un phénomène éminemment sériel ${ }^{1}$. L'idée de série est au cœur même de la problématique, résolument photogrammatique, du procédé de la prise de vues: chacune des vues n'est-elle pas constituée d'une série, d'une suite de photogrammes? La procédure du montage entretient, elle aussi, des liens assez étroits avec l'idée même de série: un film n'est-il pas constitué d'une série, d'un amalgame de plans? En même temps, l'activité cinématographique conduit souvent, dans la pratique, à l'annulation pure et simple de la sérialité constitutive du dispositif. Le rêve plus ou moins conscient de toute la génération des inventeurs de la cinématographie était non pas de produire des photogrammes (notez le pluriel), mais une série (notez, ici, le singulier) de photogrammes. La vue Edison ou la vue Lumière est d'un seul tenant: elle a beau être constituée de centaines de photographies distinctes, celle-ci sont rassemblées sur une seule bande, une bande singulière, simple et unitaire. C'est que les photogrammes, malgré leur caractère composite et multiple, ont comme fonction de donner l'illusion d'une unité et d'une continuité sans faille.

Sur le plan matériel, la cinématographie suppose donc, minimalement, la réunion de photographies distinctes que l'on dispose, mécaniquement, sur une bande pelliculaire. Sur le plan conceptuel, on a donc, d'un côté, des photogrammes multiples; de l'autre, une bande simple. D'un côté, la multiplicité, la pluralité, la diversité; de l'autre, la singularité, l'unicité, l'unité. Ce qui correspond d'ailleurs au caractère apparemment magique des vues cinématographiques: une série de photographies singu- 
lières, les photogrammes, se présentant à l'œil de l'observateur comme si elles n'étaient, en fait, qu'une seule et même photographie qui se serait animée, comme par magie.

Le dispositif cinématographique astreint donc les instances impliquées dans la production des images animées à passer de l'un (le cliché photographique, l'épreuve, le photogramme) au multiple (la multitude de clichés, la suite d'épreuves, la série de photogrammes). Il les astreint ensuite, en partant de ce multiple, à passer à cette nouvelle unité qu'est la bande pelliculaire. La mise en série de photogrammes multiples sur une bande unitaire est donc un passage de second niveau: après un passage de l'un au multiple, un passage du multiple à l'un. Ce serait ainsi, et c'est là l'hypothèse fondamentale qui se joue dans le présent article, par un constant passage dialectique, de l'un au multiple, puis de la nouvelle unité obtenue par la réunion de ces éléments multiples au multiple de cette unité, que la cinématographie aurait réussi à se développer comme dispositif.

Il faut dire que la cinématographie aurait eu de quoi tenir puisque ce serait, tout aussi bien, par un semblable processus, par une tension dialectique du même ordre, que la captation et la fixation des images photographiques elles-mêmes se seraient opérées, trois quarts de siècle avant l'invention de la cinématographie. En effet, l'intérêt de l'invention de la photographie, c'était précisément de passer du multiple au simple. Ce que les inventeurs de la photographie veulent faire, au fond, c'est d'arrêter, de capter, de fixer l'une des multiples «images virtuelles" de ce spectacle en mouvement qu'est le réel, qui se dépose, à chaque instant, à chaque minute, à chaque seconde, à chaque nanoseconde, sur le verre dépoli de la camera oscura. Du multiple, les inventeurs de la photographie veulent ne retenir que l'un; du complexe, ils veulent faire surgir le simple. Du foisonnement, ils veulent dégager quelque chose comme un "singleton" (ensemble qui est, en mathématique, formé d'un seul élément). Pour y parvenir, ils doivent de leur sujet extirper tout mouvement. L'idéal ultime des inventeurs de la photographie, c'est même d'éliminer, d'annuler, d'anéantir toute trace du mouvement qui a pu habiter cette vue se projetant sur le verre dépoli de leur «camera». Ce qui n’est pas chose facile. Prenons, 
comme exemple, cet étrange singleton qu'est la fameuse photographie de Niépce, de la perspective sur les toits, considérée comme l'une des toutes premières photographies, et qui date de 1826 ou 1827 (figure 1).

Voici donc une image qui a eu toutes les peines du monde à s'imposer ou, plutôt, à s'exposer: une douzaine d'heures de pose, à ce qu'on dit. D'où l'ombre qui porte, des deux côtés, et qui reste, à n'en point douter, une trace évidente de mouvement, de ce mouvement que font les sujets humains, et la Terre qui les supporte, dans leur course au-dessous du Soleil. Étonnant rapport au temps d'une image singulière où s'inscrit le passage, justement, du temps. En fait, et c'est la chronophotographie qui nous apprendra cela, pareille image (notez, "image" au singulier) est, en fait, une série d'images superposées (notez, ici, "images» au pluriel). C'est une image qui contient de façon non discrète cent autres images, qu'il nous est impossible de discriminer les unes des autres. Cette image, qui semble pourtant avoir les traits d'un singleton, ce serait donc

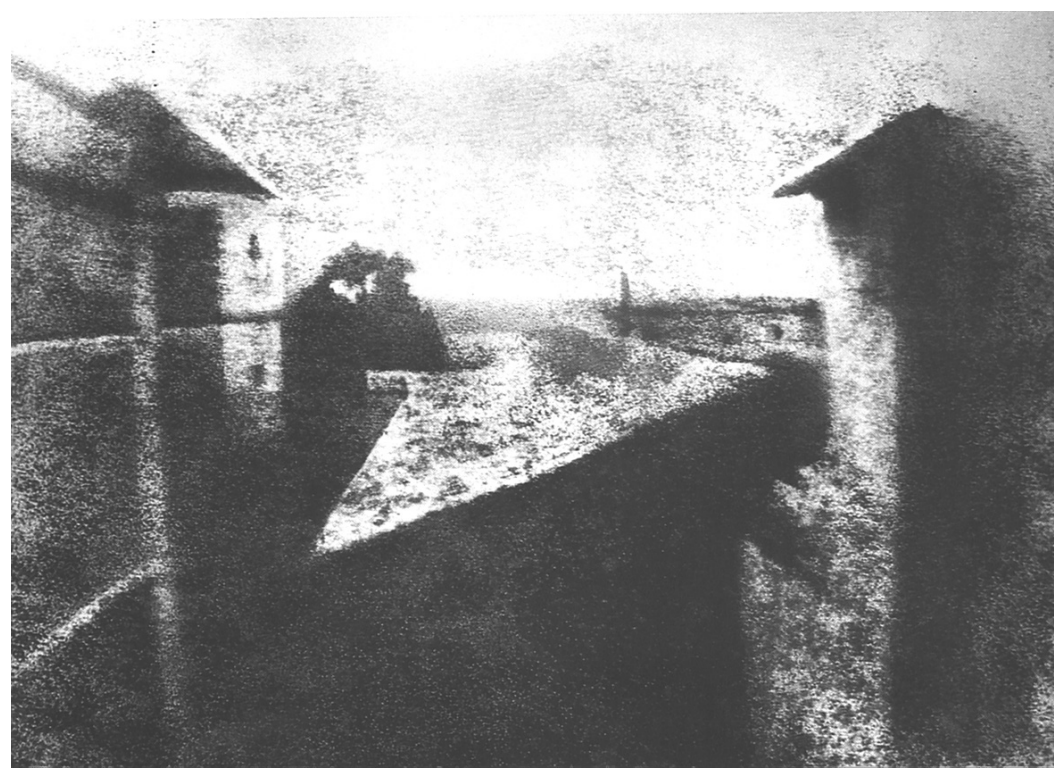

Figure 1. Perspective sur les toits, à Gras, de Nicéphore Niépce (1826). Image obtenue par un très long temps de pose (d'où l'ombre qui porte des deux côtés). 
plutôt un conglomérat de singletons indifférenciés, superposés les uns sur les autres. L'appareil photographique d'avant l'instantané, ce serait donc un peu comme un chronophotographe ou un cinématographe sans son obturateur. Ce qu'il capte, c'est bien un fragment de temps, mais un fragment qui s'inscrit dans la durée, un fragment qui inscrit la durée ${ }^{2}$. Dans l'image que l'appareil photographique d'avant l'instantané produit, il y a ainsi fusion, réunion d'une quantité de fragments temporels, la fragmentation du temps y est «indiscriminée». C'est une chronophotographie fusionnelle. On n'y a pas saucissonné le temps, comme le feront bientôt chronophotographes et cinématographistes.

En ce sens, la chronophotographie représente l'exemple canonique de la dialectique simple/multiple autour de laquelle nous gravitons ici. En tant que protocinématographie, la chronophotographie module effectivement, et de façon constante, le mouvement dialectique entre simple et multiple. C'est ce que nous apprend l'observation des plaques sensibles que produisent les expérimentations des chronophotographes. Et c'est aussi ce que nous apprend l'observation de leurs dispositifs de prise de vue. Celui de Marey, d'abord, qui met à contribution un seul appareil de prise de vues (notez, ici, le pluriel de "vues») et une plaque sensible, une seule, sur laquelle viendront s'imprimer, à partir d'un seul et unique point de vue, une série de poses successives, et multiples, d'un seul et même sujet. Celui de Muybridge, maintenant, qui met à contribution une quantité d'appareils de prise de vue (notez, ici, le singulier) et une quantité correspondante de plaques sensibles, sur chacune desquelles viendra s'imprimer, à partir d'une série de points de vue successifs, une pose, et une seule, d'un seul et même sujet.

Une caractéristique essentielle de ces dispositifs: la singularité ou la multiplicité - des appareils, des plaques, des poses ou des positions - y est chose concrète et mesurable. Ce que l'on pourra observer sur les plaques sensibles, après développement, ce seront autant de clichés qu'il y aura eu d'obturations effectives, in vivo, rien d'autre. En chronophotographie, en effet, rien ne se perd, rien ne se crée, si l'on peut dire. Il n'y a rien de 
virtuel, chacun des éléments est en correspondance biunivoque avec le modèle ayant servi de source. C'est que la chronophotographie ne ressortit finalement qu'à un simple report sur une surface sensible des impressions lumineuses, fussent-elles multiples. La chronophotographie est certes plus complexe que la simple photographie, mais cette complexité est affaire d'addition. Elle ne postule, sur le plan de la représentation, qu'un saut d'ordre quantitatif.

En cinématographie, les choses sont différentes, puisque le dispositif mis en branle permet d'accéder à un seuil d'un autre degré, à un autre ordre de représentation. Au moment de la projection, le cinématographe crée en effet une unité nouvelle, un singleton d'un ordre supérieur, produit par la conjugaison des multiples uns, des multiples singletons, par leur fusion les uns avec les autres, par l'annulation de leur singularité. C'est que, avec du fixe, le cinématographe fait du mouvement (tandis qu'avec du mouvement, la chronophotographie fait du fixe). Si, en tant qu'observateur, vous regardez le support des images, une bobine de film, et que vous vous en approchez suffisamment pour distinguer chacun des photogrammes, "What You See", au départ (= des photogrammes multiples et discontinus), "Is NOT What You Get", à l'arrivée (= une seule image continue), une fois que vous aurez placé la bande dans l'appareil et que vous aurez actionné la machine. Il y a là, de façon nettement sensible, un véritable saut qualitatif.

Ce n'est pas que la chronophotographie n'ait pas, elle aussi, un petit côté magique: elle me permet certes de voir des choses invisibles à l'œil nu, mais ce qu'elle produit, sur le plan matériel, sur le plan empirique, n'est pas un phénomène d'un ordre nouveau: ce ne sont, finalement, «que» des photographies... Des photographies multiples, certes, mais des photographies tout de même. Alors que, de son côté, la cinématographie crée, ni plus ni moins, quelque chose qui n'existe pas sur le support: l'image animée $^{3}$. Même si elle me permet seulement, au fond, de voir à nouveau ce qui était déjà visible dans le monde empirique, elle n'en fait pas moins survenir, advenir même, un phénomène d'un ordre nouveau, et qui, sur le plan photographique, n'existe qu'à l'état virtuel. C'est d'ailleurs peut-être ce qui fait que l'on peut 
considérer l'arrivée du cinématographe, dans la sphère médiatique, comme un événement de l'ordre de la rupture.

C'est ainsi, donc, que s'expliquerait l'hybridité fondamentale, la subtilité, la versatilité de ce support d'images qu'est la bande pelliculaire, située qu'elle est au croisement même de la problématique de l'un et du multiple: une bande simple et unitaire qui contient une série d'images multiples. Car les images qui composent un plan ont deux caractéristiques essentielles: elles sont multiples, mais elle sont aussi, on le sait bien, fixes, figées, arrêtées. Projeter un film, c'est projeter de multiples images fixes: La Sortie des usines Lumière, c'est une vue (unité, donc) dynamique, mais c'est aussi, il ne faut pas l'oublier, environ 800 photographies (multiplicité, donc) statiques d'ouvriers à la sortie d'une usine.

Quel paradoxe tout de même, quand on y pense, que les images animées soient faites d'images arrêtées... Quel paradoxe aussi que la sérialisation, la mise en série, soit la condition de réalisation de la mobilisation de l'image (projeter un film, c'estprojeter-de-multiples-images-fixes-sur-cette-surface-simple-qu'est-latoile-de-l'écran), mais qu'en même temps, cette mobilisation des images soit la condition d'annulation de leur sérialité (projeter un film, c'est-aussi-projeter-sur-l'écran-une-seule-image-dynamique-dumouvement-captè). Évoquer comme je le fais ici le principe de la sérialisation comme condition de réalisation de la mobilisation de l'image, c'est mettre au premier plan le fait que c'est la multiplicité des images, la multiplicité des photogrammes, qui rend possible leur animation. Évoquer le principe de la mobilisation des images comme condition d'annulation de leur sérialité, c'est mettre au premier plan le fait que, de la multiplicité des images, naît un phénomène non pas de multiplicité mais un phénomène d'unicité: la vue, le tableau, la scène, le plan, ce singleton d'un second niveau. Ainsi le multiple - un multiple lui-même constitué de singletons, les photogrammes - donne-t-il naissance au simple. Via la création d'un singleton d'une dimension nouvelle. Une dimension qui est le fruit de cette opposition thèse/antithèse, dont le jeu permet à son produit, la synthèse comme il se doit, d'accéder à un stade supérieur. Soit, le schéma suivant: 
thèse: la multiplicité photogrammatique antithèse: l'unicité pelliculaire

synthèse: la vue (qui deviendra thèse à son tour)

La thèse, c'est la multiplicité photogrammatique; l'antithèse, c'est l'unicité pelliculaire; la synthèse, c'est la vue, la vue-telleque-vue, et qui deviendra thèse à son tour, parce que le mouvement dialectique de la contradiction "simple/multiple» connaît d'autres ordres de manifestation, s'agissant du cinéma, par la procédure du montage. Mais d'abord, la conclusion de cette première partie de la présente réflexion: l'invention du procédé de la cinématographie résulte d'une "subsumption" du multiple dans l'un. Une subsumption qui, par définition, fait disparaître le subsumé dans le subsumant. Ce qui disparaît donc, au moment de la réception du film, de la vue, c'est la multiplicité photogrammatique, la sérialité originelle, cette multiplicité faite, nécessairement, d'isolats singuliers - les singletons photogrammatiques - qui, justement, disparaissent dans ce "grand tout» que devient la vue (le tableau, le plan, la scène), singleton de second niveau.

La subsumption de premier niveau est tellement centrale et essentielle en matière de cinématographie qu'elle peut mener directement à une confusion, eu égard à la pluralité et à la singularité. Prenons, par exemple, l'expression "prise de vue». L'une des subtilités de la langue française veut que, dans cette expression, le mot "vue» soit au singulier lorsqu'il est question de photographie - prise de vue — et qu'il soit au pluriel lorsqu'il est question de cinématographie — prise de vues ${ }^{4}$. Le singulier en matière de photographie, cela se comprend assez bien, mais que signifie le pluriel du mot "vues", lorsqu'il est question de cinéma? Quelles sont-elles ces «vues» multiples que l'on "prend» pour faire un film? Serait-ce parce que le cinéma est fondé sur la multiplicité des photogrammes, ces singletons de premier niveau, que le mot "vues» doit être au pluriel? Serait-ce plutôt parce que le cinéma est fondé sur la multiplicité des plans, ces singletons de deuxième niveau? 
En règle générale, on aura, bien sûr, tendance à privilégier la dernière hypothèse, puisque le mot "vue" au cinéma semble se référer au singleton de second niveau (l'unité-plan) plutôt qu'au singleton de premier niveau (l'unité-photogramme). Ainsi, lorsqu'on parle des Lumière, parle-t-on de la vue intitulée Sortie des usines. Faire un film, ce serait alors faire plusieurs vues, procéder à la "prise de vues" — littéralement —, c'est-à-dire produire de nombreux singletons de second niveau (les vues, les plans), avant d'avoir recours au montage pour les assembler, les juxtaposer. Mais on trouve aussi, du moins à l'époque des premiers temps de la cinématographie, certaines occurrences du mot "vue» pour désigner non plus les singletons de second niveau mais, bel et bien, ces singletons de premier niveau que sont les photogrammes. Rien de bien surprenant puisque, dans les autres séries culturelles dont la cinématographie s'est nourrie (la chronophotographie, la photographie, mais aussi la lanterne magique), chacune des plaques, chacune des poses, chacune des images est considérée comme une vue, de plein droit.

L’expression "prise de vues» (toujours au pluriel) se référerait alors à cette condition quasi ontologique de la cinématographicité, qui suppose la captation de multiples images, la captation de multiples "vues», par seconde. Ainsi pourrait-on avancer, en utilisant le même mot "vues" dans ses deux sens, que pour réaliser chacun des titres de leur catalogue (chacune de leurs vues donc), les Lumière ont dû prendre un peu plus d'une dizaine de vues par seconde. Voilà d'ailleurs un témoignage d'époque, qui va dans ce sens:

À l'aide d'un Kinétoscope de son invention, [M. Louis Lumière] a projeté une scène des plus curieuses: la sortie du personnel des ateliers, à l'heure du déjeuner. Cette vue animée montrant en plein mouvement tout ce monde sortant vers la rue, a produit un effet des plus saisissants [...]. Cette scène, dont le déroulement ne dure qu'une minute, ne comprend pas moins de 800 vues successives 5 .

Grâce à la magie du cinéma, les 800 poses successives de la Sortie des usines sont, à la fois et en même temps, des vues et une $v e^{6}$. La pellicule cinématographique est dotée d'une souplesse 
qui lui permet de supporter, à la fois, le singulier et le pluriel. Elle est souple, aussi, sous au moins deux autres rapports. D'abord sur un plan matériel, ensuite sur un plan conceptuel. Sur un plan matériel, parce qu'elle est constituée, physiquement, d'un produit de synthèse, le nitrate de cellulose: on peut donc, dans une certaine mesure, la plier et la replier sans la casser. Elle est souple, ensuite, sur un plan conceptuel, parce qu'elle produit une forme de synthèse. Elle produit la fameuse synthèse du mouvement mais aussi, et surtout, elle est la synthèse de l'antithèse du multiple qui s'oppose à la thèse de l'un ${ }^{7}$.

La chose la plus remarquable dans toute cette affaire, c'est que la bande souple est si souple que, tout en accueillant une manifestation évidente de sérialité, la sérialité photogrammatique, elle n'en reste pas moins, résolument, un phénomène de singularité. Singularité du tableau, singularité de la scène ou singularité du plan, ces singletons de second niveau que l'on allait bientôt mettre à leur tour en série, en passant de la vue uniponctuelle (en un seul plan) au film pluriponctuel (en plusieurs plans), en passant de la série de vues au premier sens du terme, soit la série de photogrammes, à la série de vues dans le deuxième sens, soit la série de tableaux, scènes ou plans. En effet, on passera encore une fois, en cinématographie, du simple au multiple, par le truchement d'une procédure cette fois (et non plus d'un procédé), celle du montage. Une procédure qui se développa notamment en conjuguant à son tour, depuis les tout débuts, la notion, l'idée, le concept de «série». Depuis les tout débuts parce que, comme les premières vues étaient courtes (elles ne duraient souvent pas plus d'une minute), toute séance de projection impliquait, par nécessité, la projection d'une suite, d'une succession, que dis-je, d'une série de singletons (= chacune des vues), sans nécessairement grand rapport les uns avec les autres, et presque par définition sans aucune communication les uns avec les autres. Des vues qui étaient présentées, donc, en séries plus ou moins distendues.

Il arriva aussi que les exhibiteurs opèrent des regroupements de vues en tissant quelque fil entre elles. La figure 2 (Programme Lumière au Grand Café, semaine du 3 au 10 décembre 1898) donne un exemple des deux attitudes. Les vues $\mathrm{n}^{\text {os }} 1$ et 2 sont 


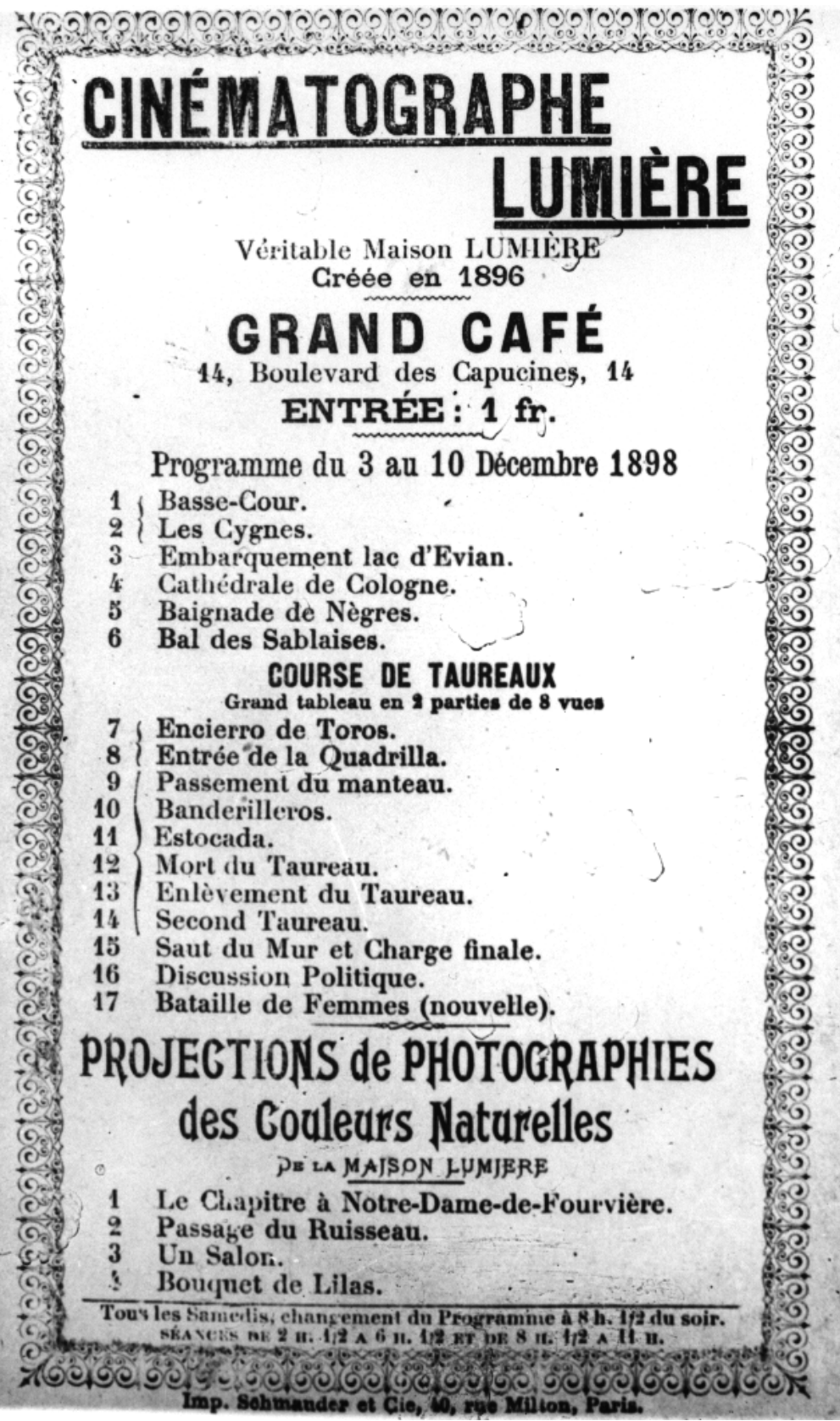

Figure 2. Programme Lumière au Grand Café, semaine du 3 au 10 décembre 1898 (Collection Institut Lumière) 
reliées l'une à l'autre par une même unité thématique. L'accolade dans la marge vient d'ailleurs souligner la chose. Suivent ensuite quatre vues disparates (les vues $\mathrm{n}^{\text {os }} 3$ à 6 ), qui n'ont aucun rapport apparent les unes avec les autres. La séance de projection se continue avec un "Grand tableau en 2 parties de 8 vues", constitué d'une première partie (délimitée par l'accolade) de deux vues (les vues $\mathrm{n}^{\text {os }} 7$ et 8 ) et d'une seconde partie de six vues (les vues 9 à 14). On souligne l'aspect non disparate de l'ensemble non seulement par des accolades, mais encore par un "sur-titre " général : "Course de taureaux.»

La constitution des séries de vues aux fins de projection était, au départ, sous l'entière responsabilité de cette instance externe à la fabrication des vues animées qu'est l'exhibiteur. Celui-ci était cependant couramment encouragé à aller dans ce sens par l'éditeur, qui regroupait les vues de son catalogue par catégories, par "séries", et y allait parfois d'indications et de suggestions explicites sur l'assemblage de certaines vues. Pour l'éditeur de vues, instance responsable du discours tenu dans les catalogues de vente, l'idée de "série» est, vraisemblablement, d'abord et avant tout une idée pratique. Il regroupe par catégories des vues relativement disparates, en les réunissant autour d'un sujet, autour d'un thème, bientôt autour d'un genre. Il s'agit alors d'aider l'exhibiteur à se retrouver dans cet amoncellement de vues qui, avec le temps, sont aussi nombreuses qu'elles sont courtes. Bien qu'elle soit l'antichambre de la pluriponctualité, la série du catalogue relève tout de même de l'uniponctualité. Il s'agit en fait d'une "uniponctualité à répétition» ou, comme on a pu le proposer ailleurs, d'une uniponctualité réitérée ${ }^{8}$.

On peut imaginer que l'idée même de "série " a, à la longue, fini par vouloir dire quelque chose pour les opérateurs euxmêmes, qui n'arrivaient pas toujours à condenser, dans la petite minute qui leur était dévolue, tout ce qu'ils avaient à montrer sur un même sujet. D'où l'idée, qui fut leur, de "couvrir» un événement en lui consacrant une série de vues. Des vues qui, dans pareil cas, avaient des liens plus étroits les unes avec les autres que dans le cas des séries disparates. La suprématie du singleton commence à être ébranlée. L'éditeur en vient alors à proposer de plus en plus souvent à l'exhibiteur 
des assemblages de vues, des assemblages préconçus, qui tiennent bien sûr plus de l'amalgame que de la concaténation. Ils n'en reposent pas moins sur un principe minimal de communication entre les vues qui les constituent. Ne serait-ce qu'en raison de la rémanence, d'un cadre à l'autre, de l'«acteur " principal (toujours le même président de la France, toujours le même tsar...), qui sert de fil rouge et qui tisse ainsi un rapport de continuité entre les vues. On n'en reste toujours pas moins pour autant sous le règne de l'uniponctualité réitérée, puisqu'il n'y a normalement, dans pareil cas, aucune communication bord à bord entre les vues.

On peut en conclure que la sérialisation par les catalogues constitua l'une des procédures qui vinrent fragiliser le règne de l'uniponctualité stricte. Comme le firent de leur côté, en contestant plus particulièrement le principe de la sacro-sainte continuité photogrammatique, ces diverses procédures de fragmentation de la bande, dont j'ai déjà fait état ailleurs ${ }^{9}$, auxquelles recouraient les premiers tourneurs de manivelle, au moment même de la prise de vues (voir plus particulièrement le procédé de la reprise, qui donna lieu, dès les débuts de la cinématographie, à une première forme de fragmentation de la bande). De fait, l'idée même de série va de pair avec la pratique de la reprise. Les deux opérations vont dans le même sens: elles luttent contre l'uniponctualité de la prise de vues et militent en faveur de l'agglomération de "fragments». Ce sont deux façons de développer l'émergence d'un singleton de troisième niveau: la séquence, le film.

Ainsi la procédure même du montage peut-elle être mise en parallèle avec le procédé de la prise de vues, puisqu'elle résulte, elle aussi, d'une subsumption du multiple dans l'un. Une subsumption qui, ici encore, fait disparaître le subsumé dans le subsumant: ce qui disparaît alors, c'est la multiplicité des plans, autre forme de sérialité originelle, une multiplicité faite, nécessairement, d'isolats singuliers - ces singletons que sont les plans - qui finissent par disparaître, précisément, dans le "grand tout ", c'est-à-dire dans ces singletons de troisième niveau que sont les séquences, les films. La mise au point de la procédure du montage résulterait donc, du moins sous la forme 


\title{
institutionnelle qu'elle a connue, d'une "subsumption" du multiple dans l'un, une subsumption de deuxième niveau.
}

\author{
Université de Montréal
}

\begin{abstract}
NOTES
1. Ce texte a été écrit dans le cadre des travaux du GRAFICS (Groupe de recherche sur l'avènement et la formation des institutions cinématographique et scénique) de l'Université de Montréal, subventionné par le Conseil de recherches en sciences humaines du Canada et le fonds FCAR du Québec. Le GRAFICS est membre du Centre de recherche sur l'intermédialité (CRI) de l'Université de Montréal. À la suite d'un colloque tenu à Udine en 2002, le présent texte a fait l'objet d'une première publication en italien, sous le titre "Dal Semplice al Multiplo o il cinema come serie di serie", dans Antonini 2003. L'auteur tient à remercier François Albera ainsi que les deux lecteurs anonymes de la revue pour leurs précieux conseils, qui l'ont amené à remanier le texte d'origine.
\end{abstract}

2. D'autant que cette soi-disant première photographie frappe notamment par l'absence dont elle témoigne de tout sujet vivant, qui n'aurait pu s'inscrire sur cette image que par un asservissement total à la négation du mouvement, qui est précisément, pour tout observateur d'une scène, le critère de passage du temps.

3. La même chose pourrait se dire du phénakistiscope, du zootrope, du praxinoscope et du kinétoscope, des mécanismes qui, à l'instar du cinématographe, font la synthèse d'une série de figures fixes en une figure unique, dotée de mouvement.

4. Voir Dournon 1966 (p. 630, article «vue»). D’où notre utilisation, plus haut, du pluriel dans le cas du dispositif de Marey, au sein duquel l'appareil est prévu pour prendre plusieurs vues, alors que le dispositif de Muybridge prévoit la prise d'une seule vue par plusieurs appareils posés côte à côte.

5. Texte apparemment anonyme écrit à la suite de la première représentation publique du Cinématographe Lumière (Société d'encouragement de l'industrie nationale), le 22 mars 1895, cité par Jacques Deslandes (1966, p. 222 — c'est moi qui souligne).

6. Sortie des usines, ce sont des vues lorsqu'on prend en considération le fait que ce film est constitué d'une bobine de pellicule remplie de clichés photographiques. Sortie des usines, c'est une vue lorsque les 800 vues sont fondues l'une dans l'autre au moment de la projection, soit lorsque la magie du cinéma opère et que l'œil perd toute fonction discriminante.

7. La bande souple est aussi un produit de synthèse sous un troisième rapport, en ceci qu'elle résout les diverses contradictions qui opposent le système-Marey au système-Muybridge. Marey, c'est la thèse: plusieurs prises de vue, discriminées les unes des autres, sur une seule plaque; Muybridge c'est l'antithèse: une succession de prises de vue, sur une pluralité de plaques, mais à chaque plaque sa prise. La bande souple Edison/Lumière (mais, aussi, toutes les bandes souples du genre, de celle du zootrope à celle sur laquelle Marey fit ses dernières expérimentations) conserve de Marey la multiplicité des prises sur une même surface, mais y rend possible une discrimination à la Muybridge.

8. Voir Gaudreault et Kessler 2002 (p. 23-32).

9. Voir notamment Gaudreault 2002 (p. 225-245). 


\section{RÉFÉRENCES BIBLIOGRAPHIQUES}

Antonini 2003: Anna Antonini (dir.), Il film e $i$ suoi multipli/Film and its Multiples, Udine, Forum, 2003.

Deslandes 1966 : Jacques Deslandes, Histoire comparée du cinéma. De la cinématique au cinématographe. 1826-1896. Tome I, Tournai, Casterman, 1966.

Dournon 1966: Jean-Yves Dournon, Le Dictionnaire des difficultés du français, Paris, Hachette, 1966.

Gaudreault 2002: André Gaudreault (collaboration: Jean-Marc Lamotte), "Fragmentation et segmentation dans les "vues animées": le corpus Lumière", dans François Albera, Marta Braun et André Gaudreault (dir.), Arrêt sur image, fragmentation du temps. Aux sources de la culture visuelle moderne/Stop Motion, Fragmentation of Time. Exploring the Roots of Modern Visual Culture, Lausanne, Payot, 2002.

Gaudreault et Kessler 2002: André Gaudreault et Frank Kessler, «L'acteur comme opérateur de continuité, ou: les aventures du corps mis en cadre, mis en scène et mis en chaîne", dans Laura Vichi (dir.), L'Uomo visible. L'attore dal cinema delle origini alle soglie del cinema moderne/The Visible Man: Film Actor from Early Cinema to the Eve of Modern Cinema, Udine, Forum, 2002. 